\title{
Numerical study of chiral symmetry breaking in non-Abelian gauge theory with background magnetic field
}

\author{
P.V. Buividovich ${ }^{\mathrm{a}, \mathrm{b}}$, M.N. Chernodub ${ }^{\mathrm{c}, \mathrm{d}, \mathrm{b}, *}$, E.V. Luschevskaya ${ }^{\mathrm{b}}$, M.I. Polikarpov $^{\mathrm{b}}$ \\ a JIPNR “Sosny”, National Academy of Science, Acad. Krasin str. 99, Minsk 220109, Belarus \\ b Institute for Theoretical and Experimental Physics, B. Cheremushkinskaya 25, Moscow 117218, Russia \\ c Laboratoire de Mathematiques et Physique Theorique, CNRS UMR 6083, Fédération Denis Poisson, Université de Tours, Parc de Grandmont, 37200, France \\ d Department of Mathematical Physics and Astronomy, University of Gent, Krijgslaan 281, S9, B-9000 Gent, Belgium
}

\section{A R T I C L E I N F O}

\section{Article history:}

Received 10 June 2009

Received in revised form 26 October 2009

Accepted 8 November 2009

Available online 13 November 2009

Editor: L. Alvarez-Gaumé

\section{PACS:}

11.30.Rd

12.38.Gc

13.40.-f

Keywords:

Quantum chromodynamics

Strong magnetic fields

Chiral symmetry breaking

\begin{abstract}
A B S T R A C T
We investigate the effect of a uniform background magnetic field on the chiral symmetry breaking in $S U(2)$ Yang-Mills theory on the lattice. We observe that the chiral condensate grows linearly with the field strength $B$ up to $\sqrt{e B}=3 \mathrm{GeV}$ as predicted by chiral perturbation theory for full QCD. As the temperature increases the coefficient in front of the linear term gets smaller. In the magnetic field nearzero eigenmodes of the Dirac operator tend to have more regular structure with larger (compared to zero-field case) Hausdorff dimensionality. We suggest that the delocalization of near-zero eigenmodes plays a crucial role in the enhancement of the chiral symmetry breaking.
\end{abstract}

(c) 2009 Elsevier B.V. All rights reserved.

\section{Introduction}

Electromagnetic interactions are most commonly used to probe various properties of other fundamental interactions, in particular, of the strong interactions which bind quarks into hadrons. In many physically relevant situations, which can be encountered, for example, in astrophysics or nuclear collisions, a reasonable approximation is that of a constant background electric or magnetic field. For instance, very strong magnetic fields $\left(B \sim 10^{16} \mathrm{~T}\right.$, $\sqrt{e B} \sim 1 \mathrm{GeV}$ ) could have been produced in the early Universe after the electroweak phase transition [1]. At the present age of the Universe, the strongest magnetic fields can probably be generated in compact dense stars, such as magnetars $\left(B \sim 10^{10} \mathrm{~T}\right.$, $\sqrt{e B} \sim 1 \mathrm{MeV}$ ) [2]. A controllable way to subject hadronic matter to a strong electromagnetic field may be provided by heavy-ion collisions in very strong laser pulses. The latter possibility will be soon realized in such experiments as PHELIX at GSI [3] and ELI [4],

\footnotetext{
* Corresponding author at: Laboratoire de Mathematiques et Physique Theorique, CNRS UMR 6083, Fédération Denis Poisson, Université de Tours, Parc de Grandmont, 37200, France. Tel.: +33 2473669 30; fax: +33 247366956 .

E-mail address: Maxim.Chernodub@lmpt.univ-tours.fr (M.N. Chernodub).
}

with estimated values of $\sqrt{e B}$ of order $0.01 \mathrm{MeV}\left(B \sim 10^{7} \mathrm{~T}\right.$ at the intensity of laser radiation $I \sim 10^{23} \mathrm{~W} / \mathrm{cm}^{2}$ ). Thus experimentally available field strengths are still much smaller than hadronic scale.

However, very strong electromagnetic fields with strength comparable to hadronic scale $\left(B \sim 10^{15} \mathrm{~T}, \sqrt{e B} \sim 300 \mathrm{MeV}\right)$ can be produced in noncentral heavy-ion collisions [5-7]. In this case, the magnetic field perpendicular to the reaction plane is due to the relative motion of the ions themselves. Such a strong magnetic field breaks $P$ and $C P$ symmetries and leads to charge separation, which manifests itself in a specific correlations between emitted positively and negatively charged particles [6,7]. Magnetic fields of the same order should also modify significantly the properties of QCD vacuum and can even change the order of the phase transition to quark-gluon plasma [8]. In particular, magnetic field couples differently to particles with different chiralities enhancing the spontaneous breaking of the chiral symmetry of $\mathrm{QCD}$, (i.e., the symmetry with respect to the interchange of massless quarks with opposite chiralities [8-13]). This phenomenon is now actively discussed in the literature $[6,8,13]$ in connection with the heavy-ion experiments at RHIC and LHC. Another interesting effect of strong external fields is the enhancement of the trace anomaly [14].

From a theorist's point of view, the effect of a strong background magnetic fields on QCD vacuum is a good test for various 
model descriptions of strong interactions. It turns out that magnetic field enhances the breaking of the chiral symmetry. A commonly used order parameter for the chiral symmetry breaking is the chiral condensate

$\Sigma \equiv-\langle 0|\bar{q} q| 0\rangle$,

which vanishes if the chiral symmetry is unbroken. If the strength of magnetic field is larger than the pion mass (which is zero for massless quarks) but is still much smaller than the hadronic scale, one can use the chiral perturbation theory to calculate the field dependence of the chiral condensate [10]. To the lowest order in the magnetic field, the chiral condensate $\Sigma(B)$ rises linearly with the field strength $B$ :

$\Sigma(B)=\Sigma(0)\left(1+\frac{e B \ln 2}{16 \pi^{2} F_{\pi}^{2}}\right)$,

where $F_{\pi} \approx 130 \mathrm{MeV}$ is the pion decay constant. The result (2) is specific to QCD: for instance, in the Nambu-Jona-Lasinio model or in AdS/QCD dual models the chiral condensate rises quadratically with the field strength $[12,13,15]$.

There are also other effects of the strong magnetic fields on the QCD vacuum. In a separate paper [16] we study the polarization of the spins of the virtual chiral quarks by the strong external field. The effect of polarization is described by the chiral magnetic susceptibility, which is related to phenomenologically interesting radiative transitions in hadron physics, to lepton pair photoproduction through specific chiral-odd coupling of a photon to quarks, and to radiative heavy meson decays [17]. The chiral magnetization of the QCD vacuum includes two effects of the strong magnetic field: the paramagnetic polarization of the quarks' spins and the enhancement of the chiral condensate. Our paper [16] is devoted to the spin effects, while the present article reports our study of the chiral enhancement.

Below we describe our results of numerical studies of chiral symmetry breaking in $S U(2)$ lattice Yang-Mills theory exposed to a uniform background magnetic field. Since non-Abelian gauge fields interact with Abelian ones only via interaction with charged quarks, in our quenched simulations we change only the Dirac operator leaving the path integral weight for non-Abelian gauge fields intact. Note that the chiral condensate in quenched theory grows logarithmically with lattice volume [18] unlike the condensate in the theory with dynamical fermions. Thus, strictly speaking, in the thermodynamic limit the chiral condensate in the quenched theory is infinite. We argue in Section 2.1 that these logarithmic corrections do not affect the slope of the linear dependence of the chiral condensate on the magnetic field (2) in the quenched limit. Thus, our result should have a smooth interpolation from the quenched theory towards full QCD.

\section{Numerical results}

\subsection{Chiral condensate}

In order to calculate the chiral condensate we use the BanksCasher formula [19], which relates the condensate (1) with the density of near-zero eigenvalues of the Dirac operator $\mathcal{D}=$ $\gamma^{\mu}\left(\partial_{\mu}-i A_{\mu}\right)$ :

$\Sigma=\lim _{\lambda \rightarrow 0} \lim _{V \rightarrow \infty} \frac{\pi \rho(\lambda)}{V}$,

where $V$ is the total four-volume of Euclidean space-time. The eigenvalues $\lambda_{n}$, the eigenmodes $\psi_{n}$ and the density of eigenvalues $\rho(\lambda)$ of the Dirac operator are defined by

$\mathcal{D} \psi_{n}=\lambda_{n} \psi_{n}, \quad \rho(\lambda)=\left\langle\sum_{n} \delta\left(\lambda-\lambda_{n}\right)\right\rangle$,

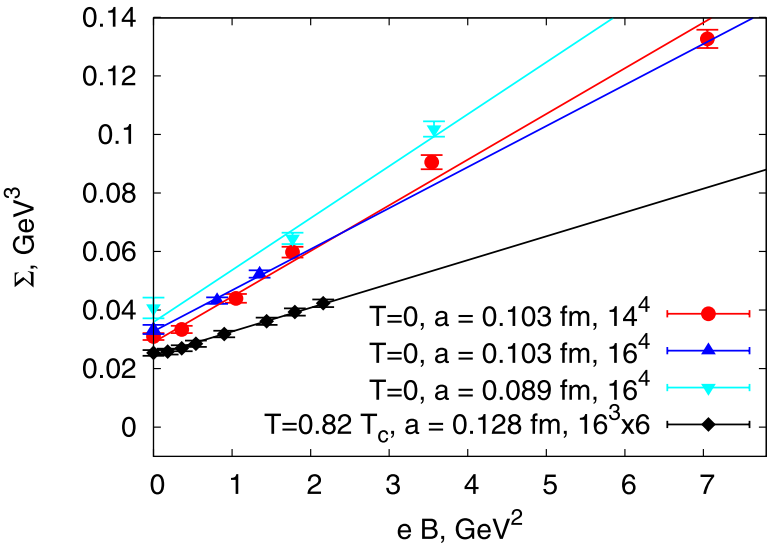

Fig. 1. The dependence of the chiral condensate on the strength of external magnetic field at different lattice volumes and lattice spacings. The solid lines are the fits by the function (7).

where in the quenched approximation the averaging is performed over the gauge fields $A_{\mu}$ with the weight $\exp \left(-S_{\mathrm{YM}}\left[A_{\mu}\right]\right)$ and $S_{\text {YM }}\left[A_{\mu}\right]$ is Yang-Mills action. In order to implement chirally symmetric massless fermions on the lattice, we use Neuberger's overlap Dirac operator [20]. Ultraviolet lattice artifacts are reduced with the help of the tadpole-improved Symanzik action for the gluon fields (see, e.g., Eq. (1) in [21]). Uniform magnetic field $B$ in the direction $\mu=3$ is introduced into the Dirac operator by substituting $s u(2)$-valued vector potential $A_{\mu}$ with $u(2)$-valued field:

$A_{\mu}^{i j} \rightarrow A_{\mu}^{i j}+A_{\mu}^{(B)} \delta^{i j}, \quad A_{\mu}^{(B)}=\frac{B}{2}\left(x_{2} \delta_{\mu 1}-x_{1} \delta_{\mu 2}\right)$.

The expression (4) is valid in the infinite volume. In order to combine it with periodic boundary conditions on the lattice we have introduced an additional $x$-dependent boundary twist for fermions [22]. In a finite hypercubic volume $L^{4}$ with periodic boundary conditions the total magnetic flux trough any twodimensional face of the hypecube is quantized [23]. The uniform magnetic field takes quantized values either,

$q B=\frac{2 \pi k}{L^{2}}, \quad k \in \mathbb{Z}$,

where

$q=\frac{1}{3} e$

is smallest (absolute value of) electric charge of the quark.

In our zero-temperature simulations we use two lattice volumes $14^{4}$ and $16^{4}$ and two lattice spacings $a=0.103 \mathrm{fm}$ and $a=$ $0.089 \mathrm{fm}$ in order to study finite-volume and finite-spacing effects. We also make simulations at nonzero temperature, $T=0.82 T_{C}$, taking the asymmetric lattice $16^{3} \times 6$. The critical temperature in $S U$ (2) gauge theory is $T_{c}=313$.(3) $\mathrm{MeV}$ [24]. The parameters of our simulations and minimal nonzero values of the magnetic field are summarized in Table 1.

Our numerical simulations reveal a gradual enhancement of the chiral condensate as the external magnetic field increases, Fig. 1. Qualitatively, this numerical result confirms various theoretical estimations [8-13].

The enhancement of the condensate can be traced already in the behavior of Dirac eigenvalues. In Figs. 2 and 3 we plot the dependence of twelve lowest Dirac eigenvalues for two typical configurations of non-Abelian gauge fields on the strength of the magnetic field. The stronger the field, the smaller eigenvalues of the Dirac operator and the larger the density of the near-zero 
Table 1

Parameters of simulations and fitting results for the chiral condensate.

\begin{tabular}{|c|c|c|c|c|c|c|c|c|c|}
\hline \multicolumn{8}{|c|}{ Parameters of simulations } & \multicolumn{2}{|c|}{ Fitting results } \\
\hline$T / T_{C}$ & $L_{S}$ & $L_{t}$ & $\beta$ & $a(\mathrm{fm})$ & $L_{s} a(\mathrm{fm})$ & $V_{4 D}\left(\mathrm{fm}^{4}\right)$ & $\sqrt{e B_{\min }}(\mathrm{MeV})$ & $\Sigma_{0}^{1 / 3}(\mathrm{MeV})$ & $\Lambda_{B}(\mathrm{GeV})$ \\
\hline 0 & 14 & 14 & 3.2810 & 0.103 & 1.44 & 4.3 & 593 & $307(15)$ & $1.36(13)$ \\
\hline 0 & 16 & 16 & 3.2810 & 0.103 & 1.65 & 7.4 & 519 & $320(5)$ & $1.53(11)$ \\
\hline 0 & 16 & 16 & 3.3555 & 0.089 & 1.42 & 4.1 & 601 & $331(19)$ & $1.43(21)$ \\
\hline 0.82 & 16 & 6 & 3.1600 & 0.128 & 2.01 & 6.6 & 418 & 291(1) & $1.74(3)$ \\
\hline
\end{tabular}

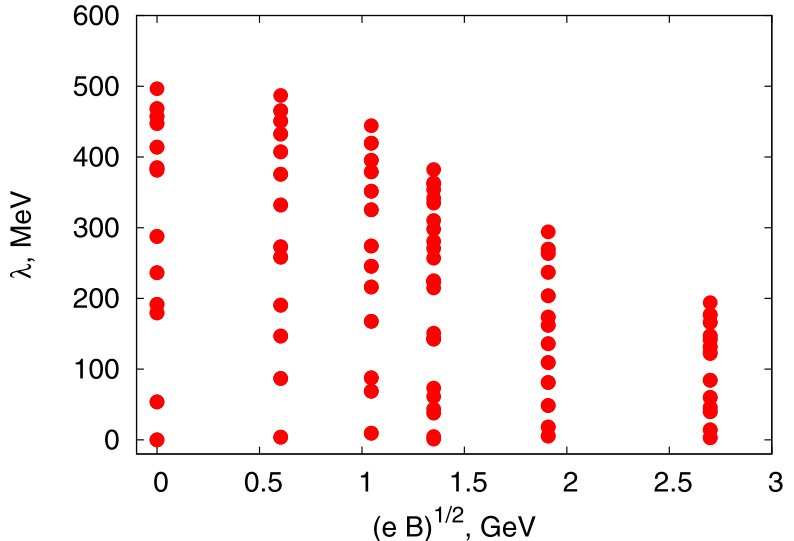

Fig. 2. The 12 lowest eigenvalues of the Dirac operator for typical configurations of non-Abelian gauge fields vs. the strength of external magnetic field for a selected configuration with unit topological charge. This configuration contains one zero Dirac eigenmode.

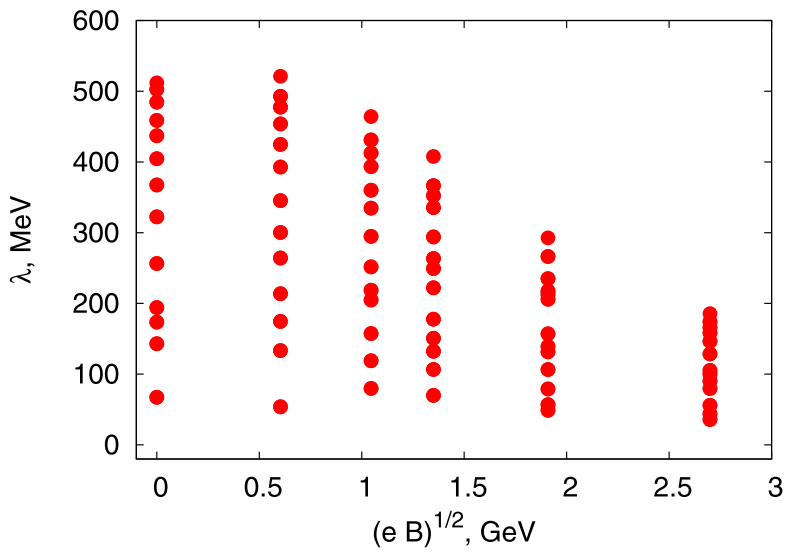

Fig. 3. The same as in Fig. 2 but for a configuration with zero topological charge (no zero Dirac eigenmodes).

modes. The later indicates the enhancement of the chiral condensate according to the Banks-Casher relation (3). Note also that the number of zero eigenvalues of the Dirac operator remain intact in external magnetic field, Fig. 2. Indeed, the Atyah-Singer theorem implies that the number of zero Dirac eigenmodes is equal to the topological charge of the gauge field configuration (we always have either left of right modes). The Abelian fields do not change the topology of the gauge fields, so that they do not influence the number of the zero modes.

We find that the dependence of the chiral condensate on the magnetic field strength can qualitatively be described by the analytical prediction (2). To this end we fit our numerical data by the linear function

$\Sigma(B)=\Sigma_{0}\left(1+\frac{e B}{\Lambda_{B}^{2}}\right)$, where $\Sigma_{0} \equiv \Sigma(0)$ and $\Lambda_{B}$ are fitting parameters. According to Fig. 1 the function (7) reproduces our data for each set of lattice parameters quite well. The best fit results are given in the last two columns of Table 1.

Before proceeding further we would like to mention a few important cautionary remarks. Firstly, our weakest magnetic fields are still quite strong as one can see from Table 1 . Indeed, the strength of the minimal magnetic field, $\left(e B_{\min }\right)^{1 / 2}$, is still greater than the scale imposed by the zero-field chiral condensate, $\Sigma_{0}^{1 / 3}$. In this regime the prediction (2) of Ref. [10] should not work, in general, as the linear behavior is expected to be realized for much weaker fields. Thus, the linear behavior is an unexpected result of our numerical simulations.

Secondly, the zero-temperature dependence of the chiral condensate $\Sigma(B)$ on the magnetic fields slightly deviates from a linear behavior at our weakest fields, as one can see in Fig. 1. Despite deviations are within error, the nonlinear features at weaker fields are not excluded by our data.

Thirdly, our largest fields are much stronger than the chiral scale, $\left(e B_{\max }\right)^{1 / 2} \ll \Sigma_{0}^{1 / 3}$. Yet, for the strongest magnetic fields that we use we do not see powerlike $\Sigma(B) \propto(e B)^{3 / 2}$ enhancement, which should be valid on dimensional grounds [10]. The reason may lie in the fact that we are studying the quenched theory, in which the virtual charged pions are absent, and the asymptotical behavior $(e B)^{3 / 2}$ may be invalid until higher energies. However, the observed linear enhancement of the chiral condensate even in the absence of the pion loops is intriguing feature of the nonAbelian gauge theory.

At zero temperature we used three different sets of lattice data to calculate the field dependence (7). The data indicates that the finite lattice spacing and finite volume corrections to our results are quite small in the studied range of parameters. The values for the zero field condensate, $\Sigma_{0}$, and for the slope parameter $\Lambda_{B}$, calculated at different lattice volumes and spacings are quite close to each other. For further reference we take the values at largest lattice with finest lattice spacing at $T=0$ :

$\Sigma_{0}^{\mathrm{fit}}=[(320 \pm 5) \mathrm{MeV}]^{3}, \quad \Lambda_{B}^{\mathrm{fit}}=(1.53 \pm 0.11) \mathrm{GeV}$.

The numerical value (8) of the chiral condensate at zero magnetic field and at zero temperature $\Sigma_{0}^{\text {fit }}$ agree well with other numerical estimations in quenched $S U(2)$ gauge theory [25]. The numerical value of the slope $\Lambda_{B}$ - also given in Eq. (8) - is relatively close to the $T=0$ result of the chiral perturbation theory (2), Ref. [10]:

$\Lambda_{B}^{\mathrm{th}}=\frac{4 \pi F_{\pi}}{\sqrt{\ln 2}}=1.97 \mathrm{GeV}$.

The later fact indicates that the quenched vacuum (studied numerically in this article) approximates the (electro)dynamics of QCD with two massless quark flavours (used in theoretical analysis of Ref. [10]) surprisingly well. Thus, the quenched vacuum can qualitatively describe the enhancement of the chiral symmetry breaking induced by the external magnetic field. Quantitatively, the quenching effects amount to be of the order to $30 \%$. 
Strictly speaking, the chiral condensate in quenched theory grows logarithmically with lattice volume [18]:

$\Sigma(V)=\Sigma_{0}\left[1+A \ln \frac{V}{V_{0}}\right]$,

where $V_{0}$ is a certain characteristic volume. Numerically, the logarithmic divergence in Eq. (10) is too small to be seen in lattice data. Indeed, in our case changing the lattice volume by a factor of two changes the chiral condensate only within the range of statistical errors (see Table 1). However, this fact raises a principal question about establishing the correspondence between our values of $\Sigma$ and $\Lambda_{B}$ and the corresponding parameters in the full theory. Numerically, we see that at our lattice volumes the chiral condensate is quite close to its value, $\Sigma^{1 / 3}=276(11)(16) \mathrm{MeV}$ in the full theory [26]. This means that the coefficient $A$ in (10) is quite small [18] and the volume $V_{0}$ is close to our lattice volumes shown in Table 1. The origin of the logarithmic volume divergence in (10) is the logarithmic divergence in the one-loop correction to pion self-energy $[10,18]$. From the Euler-Heisenberg lagrangian for a pion in external electromagnetic field [10] it is easy to see that the derivative of $\Sigma$ over $B$ does not contain this divergence. Thus, one can expect that the derivative

$\frac{\partial \Sigma(e B)}{\partial(e B)}=\frac{\Sigma_{0}}{\Lambda_{B}^{2}}$,

is finite in the infinite-volume limit. We see that actually our quenched result for the slope coefficient $\Lambda_{B}$ is quite close to the analytical prediction (9) for the full theory [10].

The finite temperature reduces both the value of the chiral condensate $\Sigma_{0}$ and increases the value of $\Lambda_{B}$, Table 1 . The last fact indicates that the linear dependence on the strength of the magnetic field gets weaker with increase of temperature.

Concluding this section we notice that in Ref. [32] the effects of the external diagonal gluonic fields on the phase diagram of QCD were studied. These fields - which are called the Abelian chromomagnetic fields - turn out to enhance the chiral condensate similarly to the external Abelian magnetic field of ordinary electromagnetism. Thus, the strong magnetic fields of gluons and the strong magnetic fields of photons have a similar influence on the condensate.

\subsection{Geometric structure of Dirac eigenmodes}

There are indications that chiral symmetry breaking is closely related with the dominance of certain singular field configurations in the vacuum of gauge theories, namely, with center vortices and Abelian monopoles [27] (for a review of monopoles and vortices see, e.g., Ref. [28]).

It has been demonstrated that removal of center vortices from gauge field configurations destroys both confinement and chiral symmetry breaking [29]. More detailed studies revealed that nearzero eigenmodes of Dirac operator, which, according to the BanksCasher formula (3), are responsible for the emergence of the chiral condensate, are strongly correlated with center vortices [30]. Similarly to center vortices, near-zero eigenmodes of Dirac operator form complex fractal structures with Hausdorff dimensionality between 2 and 3 [30,31]. Thus, it is interesting to study the effect of background magnetic fields on the localization of low-lying Dirac eigenmodes.

A commonly used measure of localization is the Inverse Participation Ratio (IPR):

$I_{n}=V \int \mathrm{d}^{4} x \rho_{n}^{2}(x) \quad$ with $\int \mathrm{d}^{4} x \rho_{n}(x)=1$.

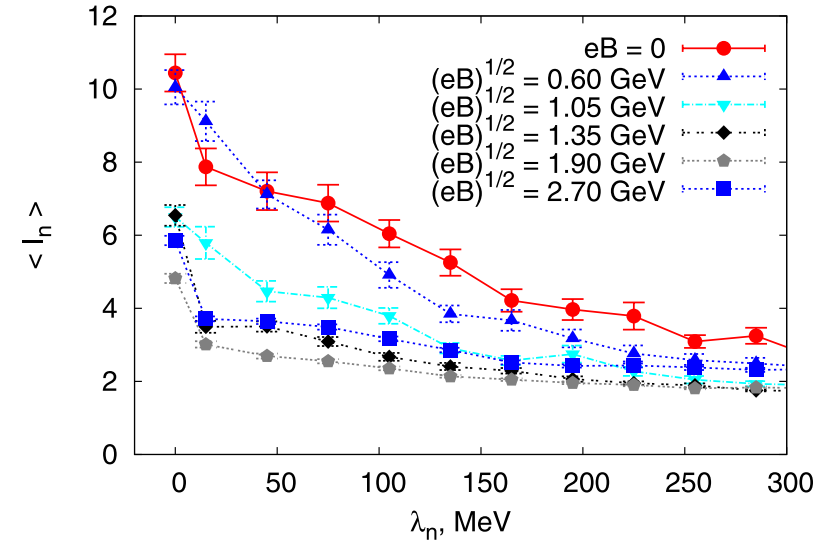

Fig. 4. IPR (12) of Dirac eigenmodes with $\lambda_{n}<500 \mathrm{MeV}$ for different strengths of the background magnetic field.
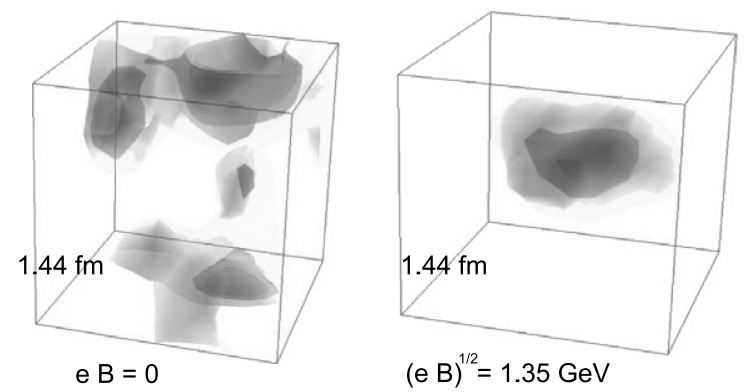

Fig. 5. Three-dimensional cuts of the density of a low-lying Dirac eigenmode for the same configuration of non-Abelian gauge fields are plotted at zero magnetic field (left) and at $\sqrt{e B}=1.35 \mathrm{GeV}$ (right).

Here $\rho_{n}(x)=\psi_{n}^{\dagger}(x) \psi_{n}(x)$ is the density of the $n$-th Dirac eigenmode and $V$ is the volume of the system.

The larger is the IPR, the more localized is the eigenmode $\psi_{n}$. For the function localized in a single point, $\psi(x) \sim \delta^{(4)}\left(x-x_{0}\right)$, the IPR is equal to the total volume of space $V$, while for a delocalized function, $\psi(x) \sim 1 / \sqrt{V}$, the IPR is equal to unity. The dependence of the IPR on the magnetic field strength and the eigenvalue $\lambda_{n}$ is illustrated in Fig. 4. For not very strong field strengths, magnetic field decreases IPR and thus delocalizes Dirac eigenmodes.

In order to visualize how the structure of the eigenmodes of the Dirac operator is modified in the external magnetic field, in Fig. 5 we have shown the three-dimensional cuts of the four-dimensional level surfaces of the densities of two near-zero Dirac eigenmodes for the same typical configuration of non-Abelian gauge fields with background magnetic field equal to zero (on the left) and with $\sqrt{e B}=1.35 \mathrm{GeV}$ (on the right). While at zero fields the eigenmode indeed has a highly irregular structure and stretches through the whole space, at $\sqrt{e B}=1.35 \mathrm{GeV}$ it occupies a compact region and has more or less smooth level surfaces. However, at zero field the IPR is still significantly higher. Why, on the one hand, the magnetic field seemingly increases the localization, and, on the other hand, decreases the IPR? This apparent paradox can be resolved if one remembers that at zero magnetic field near-zero eigenmodes of the Dirac operator are localized on lower-dimensional irregular manifolds [31], which are strongly correlated with the worldsheets of center vortices [30]. These manifolds percolate and thus fill all the physical space, in the sense that any two arbitrarily distant points have a nonzero probability to belong to the same vortex worldsheet. The total volume of these manifolds is, however, zero, and their IPR diverges as some power of ultraviolet cutoff [30,31]. 
If, on the other hand, the eigenmodes are localized in finite fourvolumes, their IPR remains finite in the continuum limit.

In a non-Abelian gauge theory only quarks interact with background Abelian fields, and in quenched theory the path-integral weight for the gauge fields $A_{\mu}$ is not modified. In particular, background fields do not affect positions of center vortices. Since the configurations of center vortices are the same for both plots in Fig. 5, we have to conclude that in background magnetic field nearzero Dirac eigenmodes become less correlated with center vortices. For sufficiently strong fields these modes are no longer associated with lower-dimensional structures with typical size set by the ultraviolet cutoff scale, but are rather localized on a physical scale determined by both hadronic scale and the strength of magnetic field.

The fact that at some large $\sqrt{e B}(\approx 2 \mathrm{GeV}$ in our case $)$ the IPR starts growing with magnetic field can be explained as follows: at this magnetic field and at finite lattice spacing the total volume of lower-dimensional manifolds on which the eigenmodes can be localized exceeds the localization volume set by the magnetic field. Then this effect should disappear in the continuum limit, $a \rightarrow 0$. One the other hand the increase of the IPR at high magnetic fields may have another simple explanation. At very strong fields the structure of the quark eigenmodes should dominantly be determined by the magnetic field and not by the underlying non-Abelian gauge field. As the magnetic field increases the quark eigenmodes tend to get localized in the transverse (to the field) directions filling out low Landau levels. The radius of the Landau levels shrinks as the magnetic field gets stronger and this effect may lead to the observed increase in the IPR. However, this effects operates at very strong gauge fields, at which the chiral condensate should presumably be determined by the scale of the magnetic field alone [10], $\Sigma \sim(e H)^{3 / 2}$.

The effect of delocalization of near-zero eigenmodes in the background magnetic field can also qualitatively explain why the chiral condensate increases with the field strength. Weaker correlation between center vortices and near-zero Dirac eigenmodes implies an increase of the total volume of space where these modes can be localized. Literally, high magnetic fields tear away the eigenmodes from the center vortices. Thus, near-zero modes become more degenerate ${ }^{1}$ and the density of near-zero eigenvalues $\rho(\lambda \rightarrow 0)$ increases, implying growth of the chiral condensate (3).

\section{Conclusions}

Summarizing, we conclude that our numerical simulations show a clear qualitative evidence that background magnetic field enhances spontaneous breaking of chiral symmetry in a nonAbelian gauge theory. We confirm that the chiral condensate rises linearly with increase of the field strength (7), in at least qualitative agreement with the prediction (2) of the chiral perturbation theory [10]. The linear dependence of the condensate on the magnetic field is governed by the parameter $\Lambda_{B}$. Surprisingly we have found that at zero temperature the value of the parameter $\Lambda_{B}$ of the quenched theory ( 8 ) is close to the analytical prediction of the chiral theory (9).

We argue that the slope of the linear dependence of the chiral condensate on the strength of the magnetic field (11) is not affected by the logarithmic volume dependence specific to the quenched limit [18].

\footnotetext{
1 In a free theory with background magnetic field near-zero modes can be localized in any point of physical space, and the chiral condensate (3) indeed diverges in the continuum limit $[9,22]$.
}

The linear dependence of the chiral condensate on the strength of the magnetic field is an unexpected result of our study because our fields are much stronger than the fields used in the analytical investigation of Ref. [10]. Moreover, the enhancement of the chiral condensate in QCD is caused by the coupling of the external magnetic field to the charged pion loops [10], which are absent in the quenched approach used in this Letter. Our result indicates that non-Abelian gauge fields alone play a significant role in the enhancement of the chiral condensate at nonzero magnetic fields. The dominance of this contribution may be revealed in further lattice simulations with dynamical fermions.

We found that the dependence of the chiral condensate on the magnetic field - encoded in the parameter $\Lambda_{B}$ - becomes much less pronounced with increase of the temperature.

We observe that the (de)localization properties of near-zero Dirac eigenmodes are crucial for the enhancement of chiral symmetry breaking in the external magnetic field. Our results suggest that the microscopic mechanism of the enhancement is the delocalization of the near-zero eigenmodes off the center vortices which increases the degeneracy of near-zero modes.

\section{Acknowledgements}

The authors are grateful to V.G. Bornyakov and S.M. Morozov for interesting discussions. This work was partly supported by grants RFBR Nos. 06-02-04010-NNIO-a, 08-02-00661-a, 06-02-17012, 0902-00629-a, and DFG-RFBR 436 RUS, BRFBR F08D-005, a grant for scientific schools Nos. NSh-679.2008.2 and NSh-4961.2008.2, by the Federal Program of the Russian Ministry of Industry, Science and Technology No. 40.052.1.1.1112, by the Russian Federal Agency for Nuclear Power, and by the STINT Institutional grant IG2004-2 025. P.V. Buividovich is also partially supported by the Euler scholarship from DAAD, by the scholarship of the "Dynasty" foundation and by the grant BRFBR F08D-005 of the Belarusian Foundation for Fundamental Research. The calculations were partially done on the MVS 50K at Moscow Joint Supercomputer Center.

\section{References}

[1] T. Vachaspati, Phys. Lett. B 265 (1991) 258.

[2] R.C. Duncan, C. Thompson, Astrophys. J. 392 (1992) L9.

[3] Petawatt high-energy laser for heavy ion experiments, http://www.gsi.de/ forschung/phelix/index_e.html.

[4] The extreme light infrastructure project (ELI), http://www.extreme-lightinfrastructure.eu/.

[5] J. Rafelski, L.P. Fulcher, A. Klein, Phys. Rep. 38 (1978) 227.

[6] D.E. Kharzeev, L.D. McLerran, H.J. Warringa, Nucl. Phys. A 803 (2008) 227, arXiv:0711.0950 [hep-ph].

[7] I.V. Selyuzhenkov, STAR Collaboration, Rom. Rep. Phys. 58 (2006) 049, arXiv:nucl-ex/0510069.

[8] N.O. Agasian, S.M. Fedorov, Phys. Lett. B 663 (2008) 445, arXiv:0803.3156 [hepph];

E.S. Fraga, A.J. Mizher, Phys. Rev. D 78 (2008) 025016, arXiv:0804.1452 [hepph];

E.S. Fraga, A.J. Mizher, Nucl. Phys. A 820 (2009) 103C, arXiv:0810.3693 [hepph].

[9] V.P. Gusynin, V.A. Miransky, I.A. Shovkovy, Phys. Lett. B 349 (1995) 477, arXiv:hep-ph/9412257.

[10] I.A. Shushpanov, A.V. Smilga, Phys. Lett. B 402 (1997) 351, arXiv:hep-ph/ 9703201.

[11] T.D. Cohen, D.A. McGady, E.S. Werbos, Phys. Rev. C 76 (2007) 055201, arXiv:0706.3208 [hep-ph].

[12] D. Ebert, K.G. Klimenko, M.A. Vdovichenko, A.S. Vshivtsev, Phys. Rev. D 61 (1999) 025005, arXiv:hep-ph/9905253.

[13] A.V. Zayakin, JHEP 0807 (2008) 116, arXiv:0807.2917 [hep-th]

[14] L. Labun, J. Rafelski, arXiv:0811.4467, arXiv:0810.1323.

[15] S.P. Klevansky, R.H. Lemmer, Phys. Rev. D 39 (1989) 3478. 
[16] P.V. Buividovich, M.N. Chernodub, E.V. Luschevskaya, M.I. Polikarpov, Nucl. Phys B 826 (2010) 313, doi:10.1016/j.nuclphysb.2009.10.008.

[17] B.L. Ioffe, A.V. Smilga, Nucl. Phys. B 232 (1984) 109;

V.M. Braun, S. Gottwald, D.Y. Ivanov, A. Schafer, L. Szymanowski, Phys. Rev. Lett. 89 (2002) 172001, arXiv:hep-ph/0206305;

B. Pire, L. Szymanowski, Phys. Rev. Lett. 103 (2009) 072002, arXiv:0905.1258 [hep-ph];

J. Rohrwild, JHEP 0709 (2007) 073, arXiv:0708.1405 [hep-ph].

[18] P.H. Damgaard, Nucl. Phys. B 608 (2001) 162, arXiv:hep-lat/0105010.

[19] T. Banks, A. Casher, Nucl. Phys. B 169 (1980) 103.

[20] H. Neuberger, Phys. Lett. B 417 (1998) 141, arXiv:hep-lat/9707022.

[21] V.G. Bornyakov, E.V. Luschevskaya, S.M. Morozov, M.I. Polikarpov, E.M. Ilgenfritz, M. Muller-Preussker, Phys. Rev. D 79 (2009) 054505, arXiv:0807.1980 [hep-lat].

[22] M.H. Al-Hashimi, U.J. Wiese, Ann. Phys. 324 (2009) 343, arXiv:0807.0630 [quant-ph].

[23] P.H. Damgaard, U.M. Heller, Nucl. Phys. B 309 (1988) 625.

[24] V.G. Bornyakov, E.M. Ilgenfritz, B.V. Martemyanov, S.M. Morozov, M. MullerPreussker, A.I. Veselov, Phys. Rev. D 76 (2007) 054505, arXiv:0706.4206 [heplat].

[25] S.J. Hands, M. Teper, Nucl. Phys. B 347 (1990) 819.
[26] C.B. Lang, P. Majumdar, W. Ortner, Phys. Lett. B 649 (2007) 225, arXiv:heplat/0611010.

[27] J. Gattnar, C. Gattringer, K. Langfeld, H. Reinhardt, A. Schafer, S. Solbrig, T. Tok, Nucl. Phys. B 716 (2005) 105, arXiv:hep-lat/0412032;

V.G. Bornyakov, E.M. Ilgenfritz, B.V. Martemyanov, S.M. Morozov, M. MullerPreussker, A.I. Veselov, Phys. Rev. D 77 (2008) 074507, arXiv:0708.3335 [heplat];

R. Hollwieser, M. Faber, J. Greensite, U.M. Heller, S. Olejnik, Phys. Rev. D 78 (2008) 054508, arXiv:0805.1846 [hep-lat].

[28] M.N. Chernodub, M.I. Polikarpov, in: P. van Baal (Ed.), Confinement, Duality, and Nonperturbative Aspects of QCD, Plenum Press, 1997, p. 387, hep-th/9710205; J. Greensite, Prog. Part. Nucl. Phys. 51 (2003) 1, arXiv:hep-lat/0301023.

[29] P. de Forcrand, M. D’Elia, Phys. Rev. Lett. 82 (1999) 4582, arXiv:hep-lat/ 9901020.

[30] M.I. Polikarpov, F.V. Gubarev, S.M. Morozov, V.I. Zakharov, PoS LAT2005 (2006) 143, arXiv:hep-lat/0510098.

[31] C. Aubin, et al., MILC Collaboration, Nucl. Phys. B (Proc. Suppl.) 140 (2005) 626, arXiv:hep-lat/0410024.

[32] P. Cea, L. Cosmai, M. D’Elia, JHEP 0712 (2007) 097, arXiv:0707.1149 [hep-lat]; L. Campanelli, M. Ruggieri, Phys. Rev. D 80 (2009) 034014, arXiv:0905.0853 [hep-ph]. 